\title{
Fifth-order generalized Heisenberg supermagnetic models
}

\author{
Nana Jiang $^{a}$, Meina Zhang ${ }^{b}$, Jiafeng Guod and Zhaowen Yand2 \\ ${ }^{a}$ School of Mathematical Sciences, Inner Mongolia University, Hohhot 010021, China \\ ${ }^{b}$ School of Mathematics and Statistics, Northeast Normal University, Changchun 130024, China \\ ${ }^{c}$ Department of Mathematical Sciences, Zibo Normal College, Zibo, 255130, China
}

\begin{abstract}
This paper is concerned with the construction of the fifth-order generalized Heisenberg supermagnetic models. We also investigate the integrable structure and properties of the supersymmetric systems. We establish their gauge equivalent equations with the gauge transformation for two quadratic constraints, i.e., the super fifth-order nonlinear Schrödinger equation and the fermionic fifth-order nonlinear Schrödinger equation, respectively.
\end{abstract}

Keywords: Heisenberg Supermagnetic Models; Gauge Transformation; Nonlinear Schrödinger Equation Mathematics Subject Classifications (2000): 17B80, 35Q55, 37K10

\section{Introduction}

The Heisenberg ferromagnet (HF) model [1, 2]

$$
\mathbf{S}_{t}=\mathbf{S} \times \mathbf{S}_{x x}, \quad \mathbf{S}=\left(S_{1}, S_{2}, S_{3}\right), \mathbf{S} \cdot \mathbf{S}=1
$$

is the simplest integrable model of ferromagnetism, where $S$ is a spin vector and $\times$ means vector product. HF model attracts a great interest mainly due to its widely applications roles in various fields in mathematics and physics, for example, the anti-de Sitter/conformal field theories [3][5], the two-dimensional gravity theory [6] and Eulerian vortex filament [7. The HF model is shown to be gauge and geometrical equivalent to the nonlinear Schrödinger equation (NLSE) [2]. In terms of the inverse scattering method, Takhtajan [8] discussed its Lax representation, the one-soliton solution and the phase and center of mass shifts for a two-soliton collision. A variety of the work has focused on the investigation of the generalized HF models, such as higher-order deformations of HF models [9, 10], the multi-component generalized HF models

\footnotetext{
${ }^{1}$ Corresponding author: jiafeng_guo@163.com

${ }^{2}$ Corresponding author: yanzw@imu.edu.cn
} 
[11, the multidimensional extended HF models [12, 13. Later on the N-soliton solutions of the generalized HF models have also been analyzed [7, 14].

Supersymmetry has its origins in quantum field theory and it receives a lot of attention [15][19]. A variety of integrable systems have been generalized into their supersymmetric equations, for instance, the Korteweg-de Vries (KdV) equation [20], the Heisenberg supermagnet (HS) model [21, 22] and the NLSE [23]. The Heisenberg supermagnetic (HS) model can be regarded as the super generalization of the HF model. Two integrable HS models are constructed on the compact subalgebra $s u(2 / 1)$. Moreover, by virtue of gauge transformation, Makhankov and Pashaev [21] proposed two types of gauge equivalent equations. It should be point out the HS model has connection with the strong electron correlated Hubbard model. Recently, a great deal of extended HS models have been studied [24, 25]. Furthermore, one [26, 27] established thirdorder and forth-order integrable HS models and presented their gauge equivalent counterparts. It is then a distinctive question to ask what is the other higher-order HS models under two constraints. When we choose a Lax operator with a function of $S$, the difficulty of construction is how to derive another Lax operator with two quadratic constraints and contains fifth-order derivatives with respect to $x$. In this paper, we intend to construct fifth-order HS models and discuss the corresponding integrability in the light of the gauge transformation.

The present paper is built up as follows. A brief review of some elementary facts with the HS model are treated in Section 2. In Section 3, we establish the fifth-order HS model and derive their equivalent equations with gauge transformation. Finally, the last Section will be devoted to a summary and discussion.

\section{Heisenberg Supermagnet Model}

The HS model can be read as 21

$$
i S_{t}=\left[S, S_{x x}\right]
$$

where $S$ is a superspin function which can be expressed as

$$
\begin{aligned}
S & =2 \sum_{a=1}^{4} S_{a} T_{a}+2 \sum_{a=5}^{8} C_{a} T_{a}, \\
& =\left(\begin{array}{ccc}
S_{3}+S_{4} & S_{1}-i S_{2} & C_{5}-i C_{6} \\
S_{1}+i S_{2} & -S_{3}+S_{4} & C_{7}-i C_{8} \\
C_{5}+i C_{6} & C_{7}+i C_{8} & 2 S_{4}
\end{array}\right),
\end{aligned}
$$

where $S_{1}, \ldots, S_{4}$ and $C_{5}, \ldots, C_{8}$ are the bosonic and fermionic variables, respectively. $T_{1}, \ldots, T_{4}$ and $T_{5}, \ldots, T_{8}$ are bosonic and fermionic generators of the superalgebra $s u(2 / 1)$, respectively.

The concept of gauge equivalence yields connections between the completely integrable systems. The most useful advantage of such interconnections is that we understand the integrable 
properties of both two systems associated with gauge transformation. It is known that the equivalent equations with gauge transformation can be embedded to their supersymmetric extensions. One proved that the HS models (2) are gauge equivalent to the flowing systems with two constraints 21]

(I). $S^{2}=S$ for $S \in S U(2 / 1) / S(L(1 / 1) \times U(1))$

$$
\begin{aligned}
& i \Phi_{t}+\Phi_{x x}+2(\Phi \bar{\Phi}+\Psi \bar{\Psi}) \Phi=0 \\
& i \Psi_{t}+\Psi_{x x}+2 \Phi \bar{\Phi} \Psi=0 .
\end{aligned}
$$

(II). $S^{2}=3 S-2 I$ for $S \in S U(2 / 1) / S(U(2) \times U(1))$

$$
\begin{aligned}
& i \Psi_{1 t}+\Psi_{1 x x}+2 \bar{\Psi}_{2} \Psi_{2} \Psi_{1}=0, \\
& i \Psi_{2 t}+\Psi_{2 x x}+2 \bar{\Psi}_{1} \Psi_{1} \Psi_{2}=0,
\end{aligned}
$$

where $\Phi(x, t)$ is a bosonic variable and $\Psi, \Psi_{1}, \Psi_{2}$ are the fermionic ones.

\section{Fifth-order Heisenberg Supermagnet Model}

Now we consider a fifth-order generalized HS model with constraint (I)

$$
S_{t}=\varepsilon S_{x x x x x}+E\left(S_{x}, S_{x x}, S_{x x x}, S_{x x x x}\right),
$$

where $\varepsilon$ is a parameter. The generalization function $E\left(S_{x}, S_{x x}, S_{x x x}, S_{x x x x}\right)$ needs to be determined which satisfies the transformation equation

$$
S E+E S=E .
$$

With the constraint (I), the superspin variable satisfies $S S_{t} S=0$ and $S\left[S, S_{x x}\right] S=0$.

Based on the Lax representation of the forth-order generalized HS model [22], the Lax pair $G$ of the fifth-order HS model contains no higher than the fourth-order derivatives with respect to $x$. Let us introduce

$$
\begin{aligned}
F & =-i \lambda S \\
G & =-i \lambda^{5} S+\lambda^{4}\left[S, S_{x}\right]+\sum_{i=1}^{n} \lambda^{i} T_{i}\left(S, S_{x}, S_{x x}, S_{x x x}, S_{x x x x}\right),
\end{aligned}
$$

where $\lambda$ is a spectral parameter.

The Lax pair satisfies the zero-curvature equation

$$
F_{t}-G_{x}+[F, G]=0
$$


Substituting (8) into (9) and using the condition (7), we obtain

$$
\begin{aligned}
E= & -5\left(S_{x x x x} S_{x}+S_{x} S_{x x x x}\right)-10\left(S_{x x x} S_{x x}+S_{x x} S_{x x x}-S S_{x x x x} S_{x}-S S_{x} S_{x x x x}\right) \\
& +15\left(S_{x} S_{x} S_{x x x}+S_{x x x} S_{x} S_{x}\right)+20\left(S_{x x} S_{x} S_{x x}+S_{x} S_{x x x} S_{x}+S S_{x x x} S_{x x}+S\right. \\
& \left.S_{x x} S_{x x x}\right)+25\left(S_{x} S_{x x} S_{x x}+S_{x x} S_{x x} S_{x}\right)+70\left(S_{x} S_{x} S_{x} S_{x} S_{x}+S S_{x x} S_{x} S_{x} S_{x}+\right. \\
& \left.S S_{x} S_{x x} S_{x} S_{x}+S S_{x} S_{x} S_{x x} S_{x}+S S_{x} S_{x} S_{x} S_{x x}\right)-35\left(S_{x x} S_{x} S_{x} S_{x}+S_{x} S_{x x} S_{x} S_{x}\right. \\
& \left.+S_{x} S_{x} S_{x x} S_{x}+S_{x} S_{x} S_{x} S_{x x}\right), \\
T_{1}= & i\left(-S_{x x x x}+5 S_{x x x} S_{x}+5 S_{x x} S_{x x}+5 S_{x} S_{x x x}-10 S S_{x x x} S_{x}-10 S S_{x x} S_{x x}-10\right. \\
& S S_{x} S_{x x x}-15 S_{x x} S_{x} S_{x}-10 S_{x} S_{x x} S_{x}-5 S_{x} S_{x} S_{x x}+35 S_{x} S_{x} S_{x} S_{x}-70 S S_{x} \\
& \left.S_{x} S_{x} S_{x}\right), \\
T_{2}= & -\left[S, S_{x x x}\right]+\left[S_{x}, S_{x x}\right]+10 S_{x} S_{x} S_{x}-20 S S_{x} S_{x} S_{x}, \\
T_{3}= & i\left(S_{x x}-3 S_{x} S_{x}+6 S S_{x} S_{x}\right), \\
T_{4}= & T_{5}=\cdots=T_{n}=0 .
\end{aligned}
$$

From the Eq.(9) and contrasting coefficients of the power of $\lambda$, we derive the higher-order HS model

$$
\begin{aligned}
i S_{t}= & i\left[S_{x x x x x}-5\left(S_{x x x} S_{x}+S_{x x} S_{x x}+S_{x} S_{x x x}-S_{x} S_{x} S_{x x}\right)_{x}+10\left(S S_{x x x} S_{x}\right.\right. \\
& \left.+S S_{x x} S_{x x}+S S_{x} S_{x x x}+S_{x} S_{x x} S_{x}\right)_{x}+15\left(S_{x x} S_{x} S_{x}\right)_{x}+35\left(2 S S_{x} S_{x} S_{x} S_{x}\right. \\
& \left.\left.-S_{x} S_{x} S_{x} S_{x}\right)_{x}\right] .
\end{aligned}
$$

The corresponding $F$ and $G$ are given by

$$
\begin{aligned}
F= & -i \lambda S, \\
G= & -i \lambda^{5} S+\lambda^{4}\left[S, S_{x}\right]+i \lambda^{3}\left(S_{x x}-3 S_{x} S_{x}+6 S S_{x} S_{x}\right)+\lambda^{2}\left(-\left[S, S_{x x x}\right]+\right. \\
& {\left.\left[S_{x}, S_{x x}\right]+10 S_{x} S_{x} S_{x}-20 S S_{x} S_{x} S_{x}\right)+i \lambda\left(-S_{x x x x}+5 S_{x x x} S_{x}+5 S_{x x}\right.} \\
& S_{x x}+5 S_{x} S_{x x x}-10 S S_{x x x} S_{x}-10 S S_{x x} S_{x x}-10 S S_{x} S_{x x x}-15 S_{x x} S_{x} S_{x} \\
& \left.-10 S_{x} S_{x x} S_{x}-5 S_{x} S_{x} S_{x x}+35 S_{x} S_{x} S_{x} S_{x}-70 S S_{x} S_{x} S_{x} S_{x}\right),
\end{aligned}
$$

where $\lambda$ is a spectral parameter.

Now we turn to consider the gauge equivalent equation of (11), let us take

$$
S(x, t)=g^{-1}(x, t) \Sigma g(x, t)
$$

where $g(x, t) \in S U(2 / 1)$.

Introducing the relation

$$
J_{1}=g_{x} g^{-1}, \quad J_{0}=g_{t} g^{-1}
$$


Eq.(14) satisfies

$$
\partial_{t} J_{1}-\partial_{x} J_{0}+\left[J_{1}, J_{0}\right]=0 .
$$

The orthogonal direct sum decomposition of the super algebra $s u(2 / 1)$ is as follows

$$
\mathrm{L}=\mathrm{L}^{(0)} \oplus \mathrm{L}^{(1)}
$$

where $\left[\mathrm{L}^{(0)}, \mathrm{L}^{(0)}\right] \subset \mathrm{L}^{(0)},\left[\mathrm{L}^{(0)}, \mathrm{L}^{(1)}\right] \subset \mathrm{L}^{(1)},\left[\mathrm{L}^{(1)}, \mathrm{L}^{(1)}\right]_{+} \subset \mathrm{L}^{(0)}$. The commutation and anticommutator relations are given by $[X, Y]=X Y-Y X,[X, Y]_{-}=X Y+Y X . L^{(0)}$ is an algebra constructed in terms of the stationary subgroup H. Suppose

$$
J_{1}=i\left(\begin{array}{ccc}
0 & \varphi & \psi \\
\bar{\varphi} & 0 & 0 \\
\bar{\psi} & 0 & 0
\end{array}\right) \in \mathrm{L}^{(1)} \text { for } S \in S U(2 / 1) / S(L(1 / 1) \times U(1))
$$

where $\varphi(x, t)$ is bosonic filed and $\psi(x, t)$ is fermionic one.

Based on (13), (14) and (17), we have

$$
\begin{aligned}
S_{t}= & g^{-1}(x, t)\left[\Sigma, J_{0}\right] g(x, t), \\
S_{x}= & g^{-1}(x, t)\left[\Sigma, J_{1}\right] g(x, t), \\
S_{x x}= & g^{-1}(x, t)\left(\left[\left[\Sigma, J_{1}\right], J_{1}\right]+\left[\Sigma, J_{1 x}\right]\right) g(x, t), \\
S_{x x x}= & g^{-1}(x, t)\left(\left[\Sigma, J_{1 x x}\right]+\left[\left[\left[\Sigma, J_{1}\right], J_{1}\right], J_{1}\right]+2\left[\left[\Sigma, J_{1 x}\right], J_{1}\right]+\left[\left[\Sigma, J_{1}\right], J_{1 x}\right]\right) \\
& g(x, t), \\
S_{x x x x}= & g^{-1}(x, t)\left(3\left[\left[\left[\Sigma, J_{1 x}\right], J_{1}\right], J_{1}\right]+3\left[\left[\Sigma, J_{1 x x}\right], J_{1}\right]+\left[\left[\left[\left[\Sigma, J_{1}\right], J_{1}\right], J_{1}\right], J_{1}\right]\right) \\
& +2\left[\left[\left[\Sigma, J_{1}\right], J_{1 x}\right], J_{1}\right]+3\left[\left[\Sigma, J_{1 x}\right], J_{1 x}\right]+\left[\Sigma, J_{1 x x x}\right]+\left[\left[\left[\Sigma, J_{1}\right], J_{1}\right], J_{1 x}\right] \\
& \left.+\left[\left[\Sigma, J_{1}\right], J_{1 x x}\right]\right) g(x, t), \\
S_{x x x x x}= & g^{-1}(x, t)\left(4\left[\left[\left[\left[\Sigma, J_{1 x}\right], J_{1}\right], J_{1}\right], J_{1}\right]+6\left[\left[\left[\Sigma, J_{1 x x}\right], J_{1}\right], J_{1}\right]\right. \\
& +\left[\left[\left[\left[\left[\Sigma, J_{1}\right], J_{1}\right], J_{1}\right], J_{1}\right], J_{1}\right]+3\left[\left[\left[\left[\Sigma, J_{1}\right], J_{1 x},\right], J_{1}\right], J_{1}\right]+8\left[\left[\left[\Sigma, J_{1 x}\right], J_{1 x}\right], J_{1}\right] \\
& +4\left[\left[\Sigma, J_{1 x x x}\right], J_{1}\right]+2\left[\left[\left[\left[\Sigma, J_{1}\right], J_{1}\right], J_{1 x}\right], J_{1}\right]+3\left[\left[\left[\Sigma, J_{1}\right], J_{1 x x}\right], J_{1}\right] \\
& +4\left[\left[\left[\Sigma, J_{1 x}\right], J_{1}\right], J_{1 x}\right]+6\left[\left[\Sigma, J_{1 x x}\right], J_{1 x}\right]+\left[\left[\left[\left[\Sigma, J_{1}\right], J_{1}\right], J_{1}\right], J_{1 x}\right] \\
& +3\left[\left[\left[\Sigma, J_{1}\right], J_{1 x}\right], J_{1 x}\right]+4\left[\left[\Sigma, J_{1 x}\right], J_{1 x x}\right]+\left[\Sigma, J_{1 x x x x}\right]+\left[\left[\left[\Sigma, J_{1}\right], J_{1}\right], J_{1 x x}\right] \\
& \left.+\left[\left[\Sigma, J_{1}\right], J_{1 x x x}\right]\right) g(x, t) .
\end{aligned}
$$

By substituting (13) and (18) into (11), we have

$$
\begin{aligned}
{\left[\Sigma, J_{0}\right]=} & 4\left[\left[\left[\left[\Sigma, J_{1 x}\right], J_{1}\right], J_{1}\right], J_{1}\right]+6\left[\left[\left[\Sigma, J_{1 x x}\right], J_{1}\right], J_{1}\right]+\left[\left[\left[\left[\left[\Sigma, J_{1}\right], J_{1}\right], J_{1}\right], J_{1}\right], J_{1}\right] \\
& +3\left[\left[\left[\left[\Sigma, J_{1}\right], J_{1 x},\right], J_{1}\right], J_{1}\right]+8\left[\left[\left[\Sigma, J_{1 x}\right], J_{1 x}\right], J_{1}\right]+4\left[\left[\Sigma, J_{1 x x x}\right], J_{1}\right] \\
& +2\left[\left[\left[\left[\Sigma, J_{1}\right], J_{1}\right], J_{1 x}\right], J_{1}\right]+3\left[\left[\left[\Sigma, J_{1}\right], J_{1 x x}\right], J_{1}\right]+4\left[\left[\left[\Sigma, J_{1 x}\right], J_{1}\right], J_{1 x}\right]
\end{aligned}
$$




$$
\begin{aligned}
& +6\left[\left[\Sigma, J_{1 x x}\right], J_{1 x}\right]+\left[\left[\left[\left[\Sigma, J_{1}\right], J_{1}\right], J_{1}\right], J_{1 x}\right]+3\left[\left[\left[\Sigma, J_{1}\right], J_{1 x}\right], J_{1 x}\right] \\
& +4\left[\left[\Sigma, J_{1 x}\right], J_{1 x x}\right]+\left[\Sigma, J_{1 x x x x}\right]+\left[\left[\left[\Sigma, J_{1}\right], J_{1}\right], J_{1 x x}\right]+\left[\left[\Sigma, J_{1}\right], J_{1 x x x}\right] \\
& -5\left(\left(\left[\Sigma, J_{1 x x}\right]+\left[\left[\left[\Sigma, J_{1}\right], J_{1}\right], J_{1}\right]+2\left[\left[\Sigma, J_{1 x}\right], J_{1}\right]+\left[\left[\Sigma, J_{1}\right], J_{1 x}\right]\right)\left[\Sigma, J_{1}\right]\right. \\
& +\left(\left[\left[\Sigma, J_{1}\right], J_{1}\right]+\left[\Sigma, J_{1 x}\right]\right)\left(\left[\left[\Sigma, J_{1}\right], J_{1}\right]+\left[\Sigma, J_{1 x}\right]\right)+\left[\Sigma, J_{1}\right]\left(\left[\Sigma, J_{1 x x}\right]\right. \\
& \left.+\left[\left[\left[\Sigma, J_{1}\right], J_{1}\right], J_{1}\right]+2\left[\left[\Sigma, J_{1 x}\right], J_{1}\right]+\left[\left[\Sigma, J_{1}\right], J_{1 x}\right]\right)-\left[\Sigma, J_{1}\right]\left[\Sigma, J_{1}\right] \\
& \left.\left(\left[\left[\Sigma, J_{1}\right], J_{1}\right]+\left[\Sigma, J_{1 x}\right]\right)\right)_{x}+10\left(\Sigma \left(\left[\Sigma, J_{1 x x}\right]+\left[\left[\left[\Sigma, J_{1}\right], J_{1}\right], J_{1}\right]\right.\right. \\
& \left.+2\left[\left[\Sigma, J_{1 x}\right], J_{1}\right]+\left[\left[\Sigma, J_{1}\right], J_{1 x}\right]\right)\left[\Sigma, J_{1}\right]+\Sigma\left(\left[\left[\Sigma, J_{1}\right], J_{1}\right]+\left[\Sigma, J_{1 x}\right]\right) \\
& \left(\left[\left[\Sigma, J_{1}\right], J_{1}\right]+\left[\Sigma, J_{1 x}\right]\right)+\Sigma\left[\Sigma, J_{1}\right]\left(\left[\Sigma, J_{1 x x}\right]+\left[\left[\left[\Sigma, J_{1}\right], J_{1}\right], J_{1}\right]\right. \\
& \left.\left.+2\left[\left[\Sigma, J_{1 x}\right], J_{1}\right]+\left[\left[\Sigma, J_{1}\right], J_{1 x}\right]\right)+\left[\Sigma, J_{1}\right]\left(\left[\left[\Sigma, J_{1}\right], J_{1}\right]+\left[\Sigma, J_{1 x}\right]\right)\left[\Sigma, J_{1}\right]\right)_{x} \\
& +15\left(\left(\left[\left[\Sigma, J_{1}\right], J_{1}\right]+\left[\Sigma, J_{1 x}\right]\right)\left[\Sigma, J_{1}\right]\left[\Sigma, J_{1}\right]\right)_{x}+35\left(2 \Sigma\left[\Sigma, J_{1}\right]\left[\Sigma, J_{1}\right]\left[\Sigma, J_{1}\right]\right. \\
& \left.\left[\Sigma, J_{1}\right]-\left[\Sigma, J_{1}\right]\left[\Sigma, J_{1}\right]\left[\Sigma, J_{1}\right]\left[\Sigma, J_{1}\right]\right)_{x},
\end{aligned}
$$

where $\Sigma=\operatorname{diag}(0,1,1)$.

From Eq.(19) and $\left[\Sigma, J_{0}^{(0)}\right]=0$, we have

$$
J_{0}^{(1)}=\left(\begin{array}{ccc}
0 & \left(J_{0}^{(1)}\right)_{12} & \left(J_{0}^{(1)}\right)_{13} \\
\left(J_{0}^{(1)}\right)_{21} & 0 & 0 \\
\left(J_{0}^{(1)}\right)_{31} & 0 & 0
\end{array}\right) \text {, }
$$

where

$$
\begin{aligned}
\left(J_{0}^{(1)}\right)_{12}= & 6 i(\varphi \bar{\varphi} \varphi \bar{\varphi} \varphi+2 \psi \bar{\psi} \varphi \bar{\varphi} \varphi)+i \varphi_{x x x x}+2 i\left(\left(\varphi \bar{\varphi}_{x}\right)_{x} \varphi+\left(\varphi_{x} \bar{\varphi}\right)_{x} \varphi+\right. \\
& \left.3\left(\varphi_{x} \varphi\right)_{x} \bar{\varphi}+2\left(\psi_{x} \varphi\right)_{x} \bar{\psi}+\left(\psi \varphi_{x}\right)_{x} \bar{\psi}+\psi\left(\bar{\psi} \varphi_{x}\right)_{x}+\left(\psi \bar{\psi}_{x}\right)_{x} \varphi\right), \\
\left(J_{0}^{(1)}\right)_{13}= & 6 i \varphi \bar{\varphi} \varphi \bar{\varphi} \psi+i \psi_{x x x x}+2 i\left(2\left(\varphi_{x} \psi\right)_{x} \bar{\varphi}+\left(\varphi \bar{\varphi}_{x}\right)_{x} \psi+\left(\bar{\varphi} \psi_{x}\right)_{x} \varphi+\right. \\
& \left.\left(\varphi \psi_{x}\right)_{x} \bar{\varphi}\right), \\
\left(J_{0}^{(1)}\right)_{21}= & 6 i(\bar{\varphi} \varphi \bar{\varphi} \varphi \bar{\varphi}+2 \bar{\varphi} \varphi \bar{\varphi} \psi \bar{\psi})+i \bar{\varphi}_{x x x x}+2 i\left(\left(\varphi_{x} \bar{\varphi}\right)_{x} \bar{\varphi}+\left(\varphi \bar{\varphi}_{x}\right)_{x} \bar{\varphi}+\right. \\
& \left.3\left(\bar{\varphi}_{x} \bar{\varphi}\right)_{x} \varphi+2 \psi\left(\bar{\varphi} \bar{\psi}_{x}\right)_{x}+\psi\left(\bar{\varphi}_{x} \bar{\psi}\right)_{x}+\left(\bar{\varphi}_{x} \psi\right)_{x} \bar{\psi}+\left(\psi_{x} \bar{\psi}\right)_{x} \bar{\varphi}\right), \\
\left(J_{0}^{(1)}\right)_{31}= & 6 i \bar{\varphi} \varphi \bar{\varphi} \varphi \bar{\psi}+i \bar{\psi}_{x x x x}+2 i\left(2\left(\bar{\varphi}_{x} \bar{\psi}\right)_{x} \varphi+\left(\bar{\varphi} \varphi_{x}\right)_{x} \bar{\psi}+\left(\varphi \bar{\psi}_{x}\right)_{x} \bar{\varphi}+\right. \\
& \left.\left(\bar{\varphi} \bar{\psi}_{x}\right)_{x} \varphi\right) .
\end{aligned}
$$

According to the Eq.(15) and Eq.(16), we obtain

$$
\left(J_{0}^{(0)}\right)_{x}=\left[J_{1}, J_{0}^{(1)}\right] .
$$

Substituting (17), (20) into (22) and integrating Eq.(22) in reference to respect to the variable $x$, we derive 


$$
J_{0}^{(0)}=\left(\begin{array}{ccc}
\left(J_{0}^{(0)}\right)_{11} & 0 & 0 \\
0 & \left(J_{0}^{(0)}\right)_{22} & \left(J_{0}^{(0)}\right)_{23} \\
0 & \left(J_{0}^{(0)}\right)_{32} & \left(J_{0}^{(0)}\right)_{33}
\end{array}\right)
$$

where

$$
\begin{aligned}
\left(J_{0}^{(0)}\right)_{11}= & 6\left(\varphi_{x} \varphi \bar{\varphi} \bar{\varphi}-\bar{\varphi}_{x} \bar{\varphi} \varphi \varphi+\varphi_{x} \psi \bar{\varphi} \bar{\psi}+\varphi \psi_{x} \bar{\varphi} \bar{\psi}-\psi \varphi \bar{\varphi}_{x} \bar{\psi}-\psi \varphi \bar{\varphi} \bar{\psi}_{x}\right) \\
& +\bar{\varphi} \varphi_{x x x}-\bar{\varphi}_{x x x} \varphi-\bar{\varphi}_{x} \varphi_{x x}+\bar{\varphi}_{x x} \varphi_{x}+\psi_{x x x} \bar{\psi}-\psi \bar{\psi}_{x x x}-\psi_{x x} \\
& \bar{\psi}_{x}+\psi_{x} \bar{\psi}_{x x}, \\
\left(J_{0}^{(0)}\right)_{22}= & 6\left(\bar{\varphi}_{x} \bar{\varphi} \varphi \varphi-\varphi_{x} \varphi \bar{\varphi} \bar{\varphi}\right)+4\left(\varphi \psi \bar{\varphi}_{x} \bar{\psi}-\varphi_{x} \psi \bar{\varphi} \bar{\psi}\right)+2\left(\varphi \psi \bar{\varphi} \bar{\psi}_{x}-\psi_{x}\right. \\
& \varphi \bar{\varphi} \bar{\psi})+\bar{\varphi}_{x x x} \varphi-\bar{\varphi} \varphi_{x x x}-\bar{\varphi}_{x x} \varphi_{x}+\bar{\varphi}_{x} \varphi_{x x}, \\
\left(J_{0}^{(0)}\right)_{23}= & 4\left(\bar{\varphi}_{x} \bar{\varphi} \varphi \psi-\varphi \psi_{x} \bar{\varphi} \bar{\varphi}\right)+2\left(\bar{\varphi}_{x} \bar{\varphi} \varphi \psi-\varphi_{x} \psi \bar{\varphi} \bar{\varphi}+\psi_{x} \bar{\psi} \bar{\varphi} \psi\right)+\bar{\varphi}_{x x x} \psi \\
& -\bar{\varphi} \psi_{x x x}-\bar{\varphi}_{x x} \psi_{x}+\bar{\varphi}_{x} \psi_{x x}, \\
\left(J_{0}^{(0)}\right)_{32}= & 4\left(\bar{\varphi} \bar{\psi}_{x} \varphi \varphi-\varphi_{x} \varphi \bar{\varphi} \bar{\psi}\right)+2\left(\bar{\varphi}_{x} \bar{\psi} \varphi \varphi-\varphi_{x} \varphi \bar{\varphi} \bar{\psi}-\bar{\psi} \varphi \psi \bar{\psi}_{x}\right)+\bar{\psi}_{x x x} \varphi \\
& -\bar{\psi} \varphi_{x x x}-\bar{\psi}_{x x} \varphi_{x}+\bar{\psi}_{x} \varphi_{x x}, \\
\left(J_{0}^{(0)}\right)_{33}= & 4\left(\bar{\varphi} \bar{\psi}_{x} \varphi \psi-\bar{\psi} \bar{\varphi} \varphi \psi_{x}\right)+2\left(\bar{\varphi}_{x} \bar{\psi} \varphi \psi-\bar{\psi} \bar{\varphi} \varphi_{x} \psi\right)+\bar{\psi}_{x x x} \psi-\bar{\psi} \psi_{x x x} \\
& -\bar{\psi}_{x x} \psi_{x}+\bar{\psi}_{x} \psi_{x x} .
\end{aligned}
$$

Since $J_{0}=J_{0}^{(0)}+J_{0}^{(1)}$, it is easy to draw the following conclusion

$$
J_{0}=\left(\begin{array}{lll}
\left(J_{0}^{(0)}\right)_{11} & \left(J_{0}^{(1)}\right)_{12} & \left(J_{0}^{(1)}\right)_{13} \\
\left(J_{0}^{(1)}\right)_{21} & \left(J_{0}^{(0)}\right)_{22} & \left(J_{0}^{(0)}\right)_{23} \\
\left(J_{0}^{(1)}\right)_{31} & \left(J_{0}^{(0)}\right)_{32} & \left(J_{0}^{(0)}\right)_{33}
\end{array}\right) .
$$

By virtue of the gauge transformation, $F$ and $G$ in (12) lead to $\hat{F}$ and $\hat{G}$, respectively.

$$
\begin{aligned}
\hat{F}= & g F g^{-1}+g_{x} g^{-1}=-i \lambda \Sigma+J_{1}, \\
\hat{G}= & g G g^{-1}+g_{t} g^{-1} \\
= & g\left(-i \lambda^{5} S+\lambda^{4}\left[S, S_{x}\right]+i \lambda^{3}\left(S_{x x}-3 S_{x} S_{x}+6 S S_{x} S_{x}\right)+\lambda^{2}\left(-\left[S, S_{x x x}\right]\right.\right. \\
& \left.+\left[S_{x}, S_{x x}\right]+10 S_{x} S_{x} S_{x}-20 S S_{x} S_{x} S_{x}\right)+i \lambda\left(-S_{x x x x}+5 S_{x x x} S_{x}+5\right. \\
& S_{x x} S_{x x}+5 S_{x} S_{x x x}-10 S S_{x x x} S_{x}-10 S S_{x x} S_{x x}-10 S S_{x} S_{x x x}-15 S_{x x} \\
& \left.S_{x} S_{x}-10 S_{x} S_{x x} S_{x}-5 S_{x} S_{x} S_{x x}+35 S_{x} S_{x} S_{x} S_{x}-70 S S_{x} S_{x} S_{x} S_{x}\right) g^{-1} \\
& +J_{0} .
\end{aligned}
$$

Substituting (17) and (25) into (26), we obtain

$$
\widehat{F}=i\left(\begin{array}{ccc}
0 & \varphi & \psi \\
\bar{\varphi} & -\lambda & 0 \\
\bar{\psi} & 0 & -\lambda
\end{array}\right), \quad \widehat{G}=\left(\begin{array}{ccc}
\widehat{G}_{11} & \widehat{G}_{12} & \widehat{G}_{13} \\
\widehat{G}_{21} & \widehat{G}_{22} & \widehat{G}_{23} \\
\widehat{G}_{31} & \widehat{G}_{32} & \widehat{G}_{33}
\end{array}\right),
$$


where

$$
\begin{aligned}
& \widehat{G}_{11}=6\left(\varphi_{x} \varphi \bar{\varphi} \bar{\varphi}-\bar{\varphi}_{x} \bar{\varphi} \varphi \varphi+\varphi_{x} \psi \bar{\varphi} \bar{\psi}+\varphi \psi_{x} \bar{\varphi} \bar{\psi}-\psi \varphi \bar{\varphi}_{x} \bar{\psi}-\psi \varphi \bar{\varphi} \bar{\psi}_{x}\right)+\bar{\varphi} \varphi_{x x x}- \\
& \bar{\varphi}_{x x x} \varphi-\bar{\varphi}_{x} \varphi_{x x}+\bar{\varphi}_{x x} \varphi_{x}+\psi_{x x x} \bar{\psi}-\psi \bar{\psi}_{x x x}-\psi_{x x} \bar{\psi}_{x}+\psi_{x} \bar{\psi}_{x x}-i \lambda^{3}(\varphi \bar{\varphi}+ \\
& \psi \bar{\psi})+\lambda^{2}\left(\varphi \bar{\varphi}_{x}+\psi \bar{\psi}_{x}-\varphi_{x} \bar{\varphi}-\psi_{x} \bar{\psi}\right)+i \lambda\left(\varphi_{x x} \bar{\varphi}+\psi_{x x} \bar{\psi}+\varphi \bar{\varphi}_{x x}+\psi \bar{\psi}_{x x}-\right. \\
& \left.\varphi_{x} \bar{\varphi}_{x}-\psi_{x} \bar{\psi}_{x}\right)+3 i \lambda \varphi \bar{\varphi} \varphi \bar{\varphi}+6 i \lambda \varphi \bar{\varphi} \psi \bar{\psi} \\
& \widehat{G}_{12}=6 i(\varphi \bar{\varphi} \varphi \bar{\varphi} \varphi+2 \psi \bar{\psi} \varphi \bar{\varphi} \varphi)+i \varphi_{x x x x}+2 i\left(\left(\varphi \bar{\varphi}_{x}\right)_{x} \varphi+\left(\varphi_{x} \bar{\varphi}\right)_{x} \varphi+3\left(\varphi_{x} \varphi\right)_{x} \bar{\varphi}+\right. \\
& \left.2\left(\psi_{x} \varphi\right)_{x} \bar{\psi}+\left(\psi \varphi_{x}\right)_{x} \bar{\psi}+\psi\left(\bar{\psi} \varphi_{x}\right)_{x}+\left(\psi \bar{\psi}_{x}\right)_{x} \varphi\right)+i \lambda^{4} \varphi+\lambda^{3} \varphi_{x}-i \lambda^{2} \varphi_{x x}- \\
& 2 i \lambda^{2}(\varphi \bar{\varphi} \varphi+\psi \bar{\psi} \varphi)-\lambda \varphi_{x x x}-6 \lambda \varphi_{x} \bar{\varphi} \varphi-3 \lambda \psi_{x} \bar{\psi} \varphi-3 \lambda \psi \bar{\psi} \varphi_{x}, \\
& \widehat{G}_{13}=6 i \varphi \bar{\varphi} \varphi \bar{\varphi} \psi+i \psi_{x x x x}+2 i\left(2\left(\varphi_{x} \psi\right)_{x} \bar{\varphi}+\left(\varphi \bar{\varphi}_{x}\right)_{x} \psi+\left(\bar{\varphi} \psi_{x}\right)_{x} \varphi+\left(\varphi \psi_{x}\right)_{x} \bar{\varphi}\right)+i \\
& \lambda^{4} \psi+\lambda^{3} \psi_{x}-i \lambda^{2} \psi_{x x}-2 i \lambda^{2} \varphi \bar{\varphi} \psi-\lambda \psi_{x x x}-3 \lambda \varphi_{x} \bar{\varphi} \psi-3 \lambda \varphi \bar{\varphi} \psi_{x}, \\
& \widehat{G}_{21}=6 i(\bar{\varphi} \varphi \bar{\varphi} \varphi \bar{\varphi}+2 \bar{\varphi} \varphi \bar{\varphi} \psi \bar{\psi})+i \bar{\varphi}_{x x x x}+2 i\left(\left(\varphi_{x} \bar{\varphi}\right)_{x} \bar{\varphi}+\left(\varphi \bar{\varphi}_{x}\right)_{x} \bar{\varphi}+3\left(\bar{\varphi}_{x} \bar{\varphi}\right)_{x} \varphi+\right. \\
& \left.2 \psi\left(\bar{\varphi} \bar{\psi}_{x}\right)_{x}+\psi\left(\bar{\varphi}_{x} \bar{\psi}\right)_{x}+\left(\bar{\varphi}_{x} \psi\right)_{x} \bar{\psi}+\left(\psi_{x} \bar{\psi}\right)_{x} \bar{\varphi}\right)+i \lambda^{4} \bar{\varphi}-\lambda^{3} \bar{\varphi}_{x}-i \lambda^{2} \bar{\varphi}_{x x}- \\
& 2 i \lambda^{2}(\bar{\varphi} \varphi \bar{\varphi}+\bar{\varphi} \psi \bar{\psi})+\lambda \bar{\varphi}_{x x x}+6 \lambda \bar{\varphi}_{x} \varphi \bar{\varphi}+3 \lambda \bar{\varphi}_{x} \psi \bar{\psi}+3 \lambda \bar{\varphi} \psi \bar{\psi}_{x} \text {, } \\
& \widehat{G}_{22}=6\left(\bar{\varphi}_{x} \bar{\varphi} \varphi \varphi-\varphi_{x} \varphi \bar{\varphi} \bar{\varphi}\right)+4\left(\varphi \psi \bar{\varphi}_{x} \bar{\psi}-\varphi_{x} \psi \bar{\varphi} \bar{\psi}\right)+2\left(\varphi \psi \bar{\varphi} \bar{\psi}_{x}-\psi_{x} \varphi \bar{\varphi} \bar{\psi}\right)+\bar{\varphi}_{x x x} \varphi \\
& -\bar{\varphi} \varphi_{x x x}-\bar{\varphi}_{x x} \varphi_{x}+\bar{\varphi}_{x} \varphi_{x x}-i \lambda^{5}+i \lambda^{3} \varphi \bar{\varphi}+\lambda^{2}\left(\bar{\varphi} \varphi_{x}-\bar{\varphi}_{x} \varphi\right)-i \lambda\left(\bar{\varphi}_{x x} \varphi+\bar{\varphi}\right. \\
& \left.\varphi_{x x}-\bar{\varphi}_{x} \varphi_{x}\right)+5 i \lambda(\bar{\varphi} \varphi \bar{\varphi} \varphi+\bar{\varphi} \psi \bar{\psi} \varphi)-8 i \lambda \bar{\varphi} \varphi \bar{\varphi} \varphi-8 i \lambda \bar{\varphi} \psi \bar{\psi} \varphi \text {, } \\
& \widehat{G}_{23}=4\left(\bar{\varphi}_{x} \bar{\varphi} \varphi \psi-\varphi \psi_{x} \bar{\varphi} \bar{\varphi}\right)+2\left(\bar{\varphi}_{x} \bar{\varphi} \varphi \psi-\varphi_{x} \psi \bar{\varphi} \bar{\varphi}+\psi_{x} \bar{\psi} \bar{\varphi} \psi\right)+\bar{\varphi}_{x x x} \psi-\bar{\varphi} \psi_{x x x}- \\
& \bar{\varphi}_{x x} \psi_{x}+\bar{\varphi}_{x} \psi_{x x}+i \lambda^{3} \bar{\varphi} \psi+\lambda^{2}\left(\bar{\varphi} \psi_{x}-\bar{\varphi}_{x} \psi\right)-i \lambda\left(\bar{\varphi}_{x x} \psi+\bar{\varphi} \psi_{x x}-\bar{\varphi}_{x} \psi_{x}\right)- \\
& 3 i \lambda \bar{\varphi} \varphi \bar{\varphi} \psi \\
& \widehat{G}_{31}=6 i \bar{\varphi} \varphi \bar{\varphi} \varphi \bar{\psi}+i \bar{\psi}_{x x x x}+2 i\left(2\left(\bar{\varphi}_{x} \bar{\psi}\right)_{x} \varphi+\left(\bar{\varphi} \varphi_{x}\right)_{x} \bar{\psi}+\left(\varphi \bar{\psi}_{x}\right)_{x} \bar{\varphi}+\left(\bar{\varphi} \bar{\psi}_{x}\right)_{x} \varphi\right)+i \lambda^{4} \\
& \bar{\psi}-\lambda^{3} \bar{\psi}_{x}-i \lambda^{2} \bar{\psi}_{x x}-2 i \lambda^{2} \bar{\psi} \varphi \bar{\varphi}+\lambda \bar{\psi}_{x x x}+3 \lambda \bar{\psi}_{x} \varphi \bar{\varphi}+3 \lambda \bar{\psi} \varphi \bar{\varphi}_{x}, \\
& \widehat{G}_{32}=4\left(\bar{\varphi} \bar{\psi}_{x} \varphi \varphi-\varphi_{x} \varphi \bar{\varphi} \bar{\psi}\right)+2\left(\bar{\varphi}_{x} \bar{\psi} \varphi \varphi-\varphi_{x} \varphi \bar{\varphi} \bar{\psi}-\bar{\psi} \varphi \psi \bar{\psi}_{x}\right)+\bar{\psi}_{x x x} \varphi-\bar{\psi} \varphi_{x x x}- \\
& \bar{\psi}_{x x} \varphi_{x}+\bar{\psi}_{x} \varphi_{x x}+i \lambda^{3} \bar{\psi} \varphi+\lambda^{2}\left(\bar{\psi} \varphi_{x}-\bar{\psi}_{x} \varphi\right)-i \lambda\left(\bar{\psi} \varphi_{x x}+\bar{\psi}_{x x} \varphi-\bar{\psi}_{x} \varphi_{x}\right)- \\
& 3 i \lambda \bar{\psi} \varphi \bar{\varphi} \varphi \text {, } \\
& \widehat{G}_{33}=4\left(\bar{\varphi} \bar{\psi}_{x} \varphi \psi-\bar{\psi} \bar{\varphi} \varphi \psi_{x}\right)+2\left(\bar{\varphi}_{x} \bar{\psi} \varphi \psi-\bar{\psi} \bar{\varphi} \varphi_{x} \psi\right)+\bar{\psi}_{x x x} \psi-\bar{\psi} \psi_{x x x}-\bar{\psi}_{x x} \psi_{x}+ \\
& \bar{\psi}_{x} \psi_{x x}-i \lambda^{5}+i \lambda^{3} \bar{\psi} \psi+\lambda^{2}\left(\bar{\psi} \psi_{x}-\bar{\psi}_{x} \psi\right)-i \lambda\left(\bar{\psi} \psi_{x x}+\bar{\psi}_{x x} \psi-\bar{\psi}_{x} \psi\right)-3 i \lambda \\
& \bar{\psi} \varphi \bar{\varphi} \psi \text {. }
\end{aligned}
$$

By means of the zero-curvature formulation of $\hat{F}$ and $\hat{G}$, we derive the super fifth-order NLSE with the constraint (I)

$$
i \varphi_{t}-6 i(\varphi \bar{\varphi} \varphi \bar{\varphi} \varphi+2 \psi \bar{\psi} \varphi \bar{\varphi} \varphi)_{x}-i \varphi_{x x x x x}-2 i\left(\left(\varphi \bar{\varphi}_{x}\right)_{x} \varphi+\left(\varphi_{x} \bar{\varphi}\right)_{x} \varphi+3\left(\varphi_{x} \varphi\right)_{x} \bar{\varphi}+\right.
$$




$$
\begin{aligned}
& \left.2\left(\psi_{x} \varphi\right)_{x} \bar{\psi}+\left(\psi \varphi_{x}\right)_{x} \bar{\psi}+\psi\left(\bar{\psi} \varphi_{x}\right)_{x}+\left(\psi \bar{\psi}_{x}\right)_{x} \varphi\right)_{x}+12 i\left(\varphi \bar{\varphi}_{x} \varphi \bar{\varphi} \varphi-\varphi_{x} \varphi \varphi \bar{\varphi} \varphi\right)-16 i \\
& \varphi_{x} \bar{\varphi} \varphi \psi \bar{\psi}+12 i\left(\varphi \bar{\varphi}_{x} \varphi \psi \bar{\psi}+\varphi \varphi \bar{\varphi} \psi \bar{\psi}_{x}\right)-8 i \varphi \varphi \bar{\varphi} \psi_{x} \bar{\psi}+i\left(2 \varphi \bar{\varphi}_{x x x} \varphi-2 \varphi \bar{\varphi} \varphi_{x x x}-2 \varphi\right. \\
& \bar{\varphi}_{x x} \varphi_{x}+2 \varphi \bar{\varphi}_{x} \varphi_{x x}+2 \psi \bar{\psi}_{x x x} \varphi-\psi \bar{\psi} \varphi_{x x x}-\psi \bar{\psi}_{x x} \varphi_{x}+\psi \bar{\psi}_{x} \varphi_{x x}-\psi_{x x x} \bar{\psi} \varphi+\psi_{x x} \bar{\psi}_{x} \\
& \left.\varphi-\psi_{x} \bar{\psi}_{x x} \varphi\right)=0, \\
& i \psi_{t}-6 i(\varphi \bar{\varphi} \varphi \bar{\varphi} \psi)_{x}-i \psi_{x x x x x}-2 i\left(2\left(\varphi_{x} \psi\right)_{x} \bar{\varphi}+\left(\varphi \bar{\varphi}_{x}\right)_{x} \psi+\left(\bar{\varphi} \psi_{x}\right)_{x} \varphi+\left(\varphi \psi_{x}\right)_{x} \bar{\varphi}\right)_{x} \\
& +12 i \varphi \bar{\varphi} \varphi \bar{\varphi}_{x} \psi-8 i \varphi_{x} \bar{\varphi} \varphi \bar{\varphi} \psi-4 i \varphi \bar{\varphi} \varphi \bar{\varphi} \psi_{x}+i\left(2 \varphi \bar{\varphi}_{x x x} \psi-\varphi \bar{\varphi} \psi_{x x x}-\varphi \bar{\varphi}_{x x} \psi_{x}+\varphi\right. \\
& \left.\bar{\varphi}_{x} \psi_{x x}-2 \psi \bar{\psi}_{x x} \psi_{x}+2 \psi \bar{\psi}_{x} \psi_{x x}-\varphi_{x x x} \bar{\varphi} \psi-\varphi_{x} \bar{\varphi}_{x x} \psi+\varphi_{x x} \bar{\varphi}_{x} \psi\right)=0 .
\end{aligned}
$$

In the following, we turn to consider the second constraint $S^{2}=3 S-2 I$. One derives $S_{t}$ and $\left[S, S_{x x}\right]$ satisfying $S S_{t} S=2 S_{t}$ and $S\left[S, S_{x x}\right] S=2\left[S, S_{x x}\right]$. Thus the deformation term $E$ should satisfy the equation

$$
S E+E S=3 E .
$$

Using the similar approach as the above constraint, we obtain the fifth-order HS model under the constraint (II)

$$
\begin{aligned}
S_{t}= & S_{x x x x x}-15\left(S_{x x x} S_{x}+S_{x x} S_{x x}+S_{x} S_{x x x}-S_{x x} S_{x} S_{x}\right)_{x}+10\left(S S_{x x x} S_{x}\right. \\
& \left.+S S_{x x} S_{x x}+S S_{x} S_{x x x}+S_{x} S_{x x} S_{x}\right)_{x}+5\left(S_{x} S_{x} S_{x x}\right)_{x}-105\left(S_{x} S_{x} S_{x} S_{x}\right)_{x} \\
& +70\left(S S_{x} S_{x} S_{x} S_{x}\right)_{x} .
\end{aligned}
$$

The corresponding $F$ and $G$ can be expressed as

$$
\begin{aligned}
F= & -i \lambda S, \\
G= & -i \lambda^{5} S+\lambda^{4}\left[S, S_{x}\right]+i \lambda^{3}\left(S_{x x}-9 S_{x} S_{x}+6 S S_{x} S_{x}\right)+\lambda^{2}\left(-\left[S, S_{x x x}\right]+\left[S_{x}, S_{x x}\right]\right. \\
& \left.+30 S_{x} S_{x} S_{x}-20 S S_{x} S_{x} S_{x}\right)+i \lambda\left(-S_{x x x x}+15 S_{x x x} S_{x}+15 S_{x x} S_{x x}+15 S_{x} S_{x x x}\right. \\
& -10 S S_{x x x} S_{x}-10 S S_{x x} S_{x x}-10 S S_{x} S_{x x x}-15 S_{x x} S_{x} S_{x}-10 S_{x} S_{x x} S_{x}-5 S_{x} S_{x} \\
& \left.S_{x x}+105 S_{x} S_{x} S_{x} S_{x}-70 S S_{x} S_{x} S_{x} S_{x}\right),
\end{aligned}
$$

where $\lambda$ is the spectral parameter.

Let

$$
J_{1}=i\left(\begin{array}{ccc}
0 & 0 & \psi_{1} \\
0 & 0 & \psi_{2} \\
\bar{\psi}_{1} & \bar{\psi}_{2} & 0
\end{array}\right) \in \mathrm{L}^{(1)} \text { for } S \in S U(2 / 1) / S(U(2) \times U(1))
$$

here $\psi_{1}(x, t), \psi_{2}(x, t)$ are the fermionic variables. 
Substituting (13) and (18) into (31), we find

$$
\begin{aligned}
{\left[\Sigma, J_{0}\right]=} & 4\left[\left[\left[\left[\Sigma, J_{1 x}\right], J_{1}\right], J_{1}\right], J_{1}\right]+6\left[\left[\left[\Sigma, J_{1 x x}\right], J_{1}\right], J_{1}\right]+\left[\left[\left[\left[\left[\Sigma, J_{1}\right], J_{1}\right], J_{1}\right], J_{1}\right], J_{1}\right] \\
& +3\left[\left[\left[\left[\Sigma, J_{1}\right], J_{1 x},\right], J_{1}\right], J_{1}\right]+8\left[\left[\left[\Sigma, J_{1 x}\right], J_{1 x}\right], J_{1}\right]+4\left[\left[\Sigma, J_{1 x x x}\right], J_{1}\right] \\
& +2\left[\left[\left[\left[\Sigma, J_{1}\right], J_{1}\right], J_{1 x}\right], J_{1}\right]+3\left[\left[\left[\Sigma, J_{1}\right], J_{1 x x}\right], J_{1}\right]+4\left[\left[\left[\Sigma, J_{1 x}\right], J_{1}\right], J_{1 x}\right] \\
& +6\left[\left[\Sigma, J_{1 x x}\right], J_{1 x}\right]+\left[\left[\left[\left[\Sigma, J_{1}\right], J_{1}\right], J_{1}\right], J_{1 x}\right]+3\left[\left[\left[\Sigma, J_{1}\right], J_{1 x}\right], J_{1 x}\right] \\
& +4\left[\left[\Sigma, J_{1 x}\right], J_{1 x x}\right]+\left[\Sigma, J_{1 x x x x}\right]+\left[\left[\left[\Sigma, J_{1}\right], J_{1}\right], J_{1 x x}\right]+\left[\left[\Sigma, J_{1}\right], J_{1 x x x}\right] \\
& -15\left(\left(\left[\Sigma, J_{1 x x}\right]+\left[\left[\left[\Sigma, J_{1}\right], J_{1}\right], J_{1}\right]+2\left[\left[\Sigma, J_{1 x}\right], J_{1}\right]+\left[\left[\Sigma, J_{1}\right], J_{1 x}\right]\right)\left[\Sigma, J_{1}\right]\right. \\
& +\left(\left[\left[\Sigma, J_{1}\right], J_{1}\right]+\left[\Sigma, J_{1 x}\right]\right)\left(\left[\left[\Sigma, J_{1}\right], J_{1}\right]+\left[\Sigma, J_{1 x}\right]\right)+\left[\Sigma, J_{1}\right]\left(\left[\Sigma, J_{1 x x}\right]\right. \\
& \left.+\left[\left[\left[\Sigma, J_{1}\right], J_{1}\right], J_{1}\right]+2\left[\left[\Sigma, J_{1 x}\right], J_{1}\right]+\left[\left[\Sigma, J_{1}\right], J_{1 x}\right]\right)-\left(\left[\left[\Sigma, J_{1}\right], J_{1}\right]+\left[\Sigma, J_{1 x}\right]\right) \\
& {\left.\left[\Sigma, J_{1}\right]\left[\Sigma, J_{1}\right]\right)_{x}+10\left(\Sigma \left(\left[\Sigma, J_{1 x x}\right]+\left[\left[\left[\Sigma, J_{1}\right], J_{1}\right], J_{1}\right]+2\left[\left[\Sigma, J_{1 x}\right], J_{1}\right]\right.\right.} \\
& \left.+\left[\left[\Sigma, J_{1}\right], J_{1 x}\right]\right)\left[\Sigma, J_{1}\right]+\Sigma\left(\left[\left[\Sigma, J_{1}\right], J_{1}\right]+\left[\Sigma, J_{1 x}\right]\right)\left(\left[\left[\Sigma, J_{1}\right], J_{1}\right]+\left[\Sigma, J_{1 x}\right]\right) \\
& +\Sigma\left[\Sigma, J_{1}\right]\left(\left[\Sigma, J_{1 x x}\right]+\left[\left[\left[\Sigma, J_{1}\right], J_{1}\right], J_{1}\right]+2\left[\left[\Sigma, J_{1 x}\right], J_{1}\right]+\left[\left[\Sigma, J_{1}\right], J_{1 x}\right]\right) \\
& \left.+\left[\Sigma, J_{1}\right]\left(\left[\left[\Sigma, J_{1}\right], J_{1}\right]+\left[\Sigma, J_{1 x}\right]\right)\left[\Sigma, J_{1}\right]\right)_{x}+5\left([ \Sigma , J _ { 1 } ] [ \Sigma , J _ { 1 } ] \left(\left[\left[\Sigma, J_{1}\right], J_{1}\right]\right.\right. \\
& \left.\left.+\left[\Sigma, J_{1 x}\right]\right)\right)_{x}-105\left(\left[\Sigma, J_{1}\right]\left[\Sigma, J_{1}\right]\left[\Sigma, J_{1}\right]\left[\Sigma, J_{1}\right]\right)_{x}+70\left(\Sigma\left[\Sigma, J_{1}\right]\left[\Sigma, J_{1}\right]\right. \\
& {\left.\left[\Sigma, J_{1}\right]\left[\Sigma, J_{1}\right]\right)_{x}, }
\end{aligned}
$$

where $\Sigma=\operatorname{diag}(1,1,2)$.

Repeating the process of constraint I, naturally, we obtain

$$
J_{0}^{(0)}=\left(\begin{array}{ccc}
\left(J_{0}^{(0)}\right)_{11} & \left(J_{0}^{(0)}\right)_{12} & 0 \\
\left(J_{0}^{(0)}\right)_{21} & \left(J_{0}^{(0)}\right)_{22} & 0 \\
0 & 0 & \left(J_{0}^{(0)}\right)_{33}
\end{array}\right), \quad J_{0}^{(1)}=\left(\begin{array}{ccc}
0 & 0 & \left(J_{0}^{(1)}\right)_{13} \\
0 & 0 & \left(J_{0}^{(1)}\right)_{23} \\
\left(J_{0}^{(1)}\right)_{31} & \left(J_{0}^{(1)}\right)_{32} & 0
\end{array}\right) \text {. }
$$

Combining the two matrix in (35), we obtain

$$
J_{0}=\left(\begin{array}{lll}
\left(J_{0}^{(0)}\right)_{11} & \left(J_{0}^{(0)}\right)_{12} & \left(J_{0}^{(1)}\right)_{13} \\
\left(J_{0}^{(0)}\right)_{21} & \left(J_{0}^{(0)}\right)_{22} & \left(J_{0}^{(1)}\right)_{23} \\
\left(J_{0}^{(1)}\right)_{31} & \left(J_{0}^{(1)}\right)_{32} & \left(J_{0}^{(0)}\right)_{33}
\end{array}\right),
$$

where

$$
\begin{aligned}
\left(J_{0}^{(0)}\right)_{11}= & 4\left(\psi_{1} \psi_{2} \bar{\psi}_{2} \bar{\psi}_{1 x}-\psi_{1 x} \psi_{2} \bar{\psi}_{2} \bar{\psi}_{1}\right)-2\left(\bar{\psi}_{2} \bar{\psi}_{1} \psi_{1} \psi_{2 x}-\psi_{1} \psi_{2} \bar{\psi}_{2 x} \bar{\psi}_{1}\right)+ \\
& \psi_{1 x x x} \bar{\psi}_{1}-\psi_{1} \bar{\psi}_{1 x x x}-\psi_{1 x x} \bar{\psi}_{1 x}+\psi_{1 x} \bar{\psi}_{1 x x} \\
\left(J_{0}^{(0)}\right)_{12}= & 2\left(\psi_{1} \bar{\psi}_{2 x} \psi_{2} \bar{\psi}_{2}-\psi_{1} \bar{\psi}_{1} \psi_{1 x} \bar{\psi}_{2}\right)+\psi_{1 x x x} \bar{\psi}_{2}-\psi_{1} \bar{\psi}_{2 x x x}-\psi_{1 x x} \bar{\psi}_{2 x} \\
& +\psi_{1 x} \bar{\psi}_{2 x x} \\
\left(J_{0}^{(1)}\right)_{13}= & -4 i\left(\psi_{1 x} \psi_{2}\right)_{x} \bar{\psi}_{2}-2 i\left(\bar{\psi}_{2}\left(\psi_{1} \psi_{2 x}\right)_{x}+\psi_{1}\left(\psi_{2 x} \bar{\psi}_{2}\right)_{x}-\left(\psi_{1} \bar{\psi}_{2 x}\right)_{x} \psi_{2}\right) \\
& +i \psi_{1 x x x x}
\end{aligned}
$$




$$
\begin{aligned}
\left(J_{0}^{(0)}\right)_{21}= & 2\left(\psi_{2} \bar{\psi}_{1 x} \psi_{1} \bar{\psi}_{1}-\psi_{2} \bar{\psi}_{2} \psi_{2 x} \bar{\psi}_{1}\right)+\psi_{2 x x x} \bar{\psi}_{1}-\psi_{2} \bar{\psi}_{1 x x x}-\psi_{2 x x} \bar{\psi}_{1 x} \\
& +\psi_{2 x} \bar{\psi}_{1 x x}, \\
\left(J_{0}^{(0)}\right)_{22}= & 4\left(\psi_{2} \bar{\psi}_{1} \bar{\psi}_{2 x} \psi_{1}-\psi_{2 x} \psi_{1} \bar{\psi}_{1} \bar{\psi}_{2}\right)-2\left(\bar{\psi}_{1} \psi_{2} \psi_{1 x} \bar{\psi}_{2}-\psi_{2} \bar{\psi}_{1 x} \bar{\psi}_{2} \psi_{1}\right)+ \\
& \psi_{2 x x x} \bar{\psi}_{2}-\psi_{2} \bar{\psi}_{2 x x x}-\psi_{2 x x} \bar{\psi}_{2 x}+\psi_{2 x} \bar{\psi}_{2 x x}, \\
\left(J_{0}^{(1)}\right)_{23}= & -4 i\left(\psi_{2 x} \psi_{1}\right)_{x} \bar{\psi}_{1}-2 i\left(\bar{\psi}_{1}\left(\psi_{2} \psi_{1 x}\right)_{x}-\psi_{2}\left(\bar{\psi}_{1} \psi_{1 x}\right)_{x}-\left(\psi_{2} \bar{\psi}_{1 x}\right)_{x} \psi_{1}\right) \\
& +i \psi_{2 x x x x}, \\
\left(J_{0}^{(1)}\right)_{31}= & -4 i\left(\bar{\psi}_{2} \bar{\psi}_{1 x}\right)_{x} \psi_{2}+2 i\left(\bar{\psi}_{1}\left(\bar{\psi}_{2 x} \psi_{2}\right)_{x}+\bar{\psi}_{2}\left(\psi_{2 x} \bar{\psi}_{1}\right)_{x}-\left(\bar{\psi}_{2 x} \bar{\psi}_{1}\right)_{x} \psi_{2}\right) \\
& +i \bar{\psi}_{1 x x x x}, \\
\left(J_{0}^{(1)}\right)_{32}= & -4 i\left(\bar{\psi}_{1} \bar{\psi}_{2 x}\right)_{x} \psi_{1}+2 i\left(\bar{\psi}_{2}\left(\bar{\psi}_{1 x} \psi_{1}\right)_{x}+\bar{\psi}_{1}\left(\psi_{1 x} \bar{\psi}_{2}\right)_{x}-\left(\bar{\psi}_{1 x} \bar{\psi}_{2}\right)_{x} \psi_{1}\right) \\
& +i \bar{\psi}_{2 x x x x}, \\
\left(J_{0}^{(0)}\right)_{33}= & 6\left(\bar{\psi}_{1} \psi_{1 x} \psi_{2} \bar{\psi}_{2}+\bar{\psi}_{2} \psi_{2 x} \psi_{1} \bar{\psi}_{1}-\bar{\psi}_{2} \bar{\psi}_{1 x} \psi_{2} \psi_{1}-\bar{\psi}_{1} \bar{\psi}_{2 x} \psi_{1} \psi_{2}\right)+\bar{\psi}_{1 x x x} \\
& \psi_{1}-\bar{\psi}_{1} \psi_{1 x x x}-\bar{\psi}_{1 x x} \psi_{1 x}+\bar{\psi}_{1 x} \psi_{1 x x}+\bar{\psi}_{2 x x x} \psi_{2}-\bar{\psi}_{2} \psi_{2 x x x}-\bar{\psi}_{2 x x} \\
& \psi_{2 x}+\bar{\psi}_{2 x} \psi_{2 x x} .
\end{aligned}
$$

By virtue of the gauge transformation, $F$ and $G(32)$ lead to $\tilde{F}$ and $\tilde{G}$

$$
\begin{aligned}
\tilde{F}= & g U g^{-1}+g_{x} g^{-1}=-i \lambda \Sigma+J_{1}, \\
\tilde{G}= & g V g^{-1}+g_{t} g^{-1} \\
= & g\left(-i \lambda^{5} S+\lambda^{4}\left[S, S_{x}\right]+i \lambda^{3}\left(S_{x x}-3 S_{x} S_{x}+6 S S_{x} S_{x}\right)+\lambda^{2}\left(-\left[S, S_{x x x}\right]\right.\right. \\
& \left.+\left[S_{x}, S_{x x}\right]+10 S_{x} S_{x} S_{x}-20 S S_{x} S_{x} S_{x}\right)+i \lambda\left(-S_{x x x x}+5 S_{x x x} S_{x}+5\right. \\
& S_{x x} S_{x x}+5 S_{x} S_{x x x}-10 S S_{x x x} S_{x}-10 S S_{x x} S_{x x}-10 S S_{x} S_{x x x}-15 S_{x x} \\
& \left.\left.S_{x} S_{x}-10 S_{x} S_{x x} S_{x}-5 S_{x} S_{x} S_{x x}+35 S_{x} S_{x} S_{x} S_{x}-70 S S_{x} S_{x} S_{x} S_{x}\right)\right) g^{-1} \\
& +J_{0} .
\end{aligned}
$$

By means of (33) and (36), we rewrite (38) as follows

$$
\tilde{F}=i\left(\begin{array}{ccc}
-\lambda & 0 & \psi_{1} \\
0 & -\lambda & \psi_{2} \\
\bar{\psi}_{1} & \bar{\psi}_{2} & -2 \lambda
\end{array}\right), \quad \tilde{G}=\left(\begin{array}{ccc}
\tilde{G}_{11} & \tilde{G}_{12} & \tilde{G}_{13} \\
\tilde{G}_{21} & \tilde{G}_{22} & \tilde{G}_{23} \\
\tilde{G}_{31} & \tilde{G}_{32} & \tilde{G}_{33}
\end{array}\right),
$$

where

$$
\begin{aligned}
\tilde{G}_{11}= & -i \lambda^{5}-i \lambda^{3} \psi_{1} \bar{\psi}_{1}-\lambda^{2}\left(\psi_{1 x} \bar{\psi}_{1}-\psi_{1} \bar{\psi}_{1 x}\right)+i \lambda\left(\psi_{1} \bar{\psi}_{1 x x}+\psi_{1 x x} \bar{\psi}_{1}\right) \\
& -i \lambda \psi_{1 x} \bar{\psi}_{1 x}+3 i \lambda \psi_{1} \bar{\psi}_{2} \psi_{2} \bar{\psi}_{1}+4\left(\psi_{1} \psi_{2} \bar{\psi}_{2} \bar{\psi}_{1 x}-\psi_{1 x} \psi_{2} \bar{\psi}_{2} \bar{\psi}_{1}\right)-2 \\
& \left(\bar{\psi}_{2} \bar{\psi}_{1} \psi_{1} \psi_{2 x}-\psi_{1} \psi_{2} \bar{\psi}_{2 x} \bar{\psi}_{1}\right)+\left(\psi_{1 x x x} \bar{\psi}_{1}-\psi_{1} \bar{\psi}_{1 x x x}-\psi_{1 x x} \bar{\psi}_{1 x}+\right. \\
& \left.\psi_{1 x} \bar{\psi}_{1 x x}\right),
\end{aligned}
$$




$$
\begin{aligned}
& \tilde{G}_{12}=-i \lambda^{3} \psi_{1} \bar{\psi}_{2}-\lambda^{2}\left(\psi_{1 x} \bar{\psi}_{2}-\psi_{1} \bar{\psi}_{2 x}\right)+i \lambda\left(\psi_{1 x x} \bar{\psi}_{2}+\psi_{1} \bar{\psi}_{2 x x}\right)-i \lambda \psi_{1 x} \\
& \bar{\psi}_{2 x}+2\left(\psi_{1} \bar{\psi}_{2 x} \psi_{2} \bar{\psi}_{2}-\psi_{1} \bar{\psi}_{1} \psi_{1 x} \bar{\psi}_{2}\right)+\left(\psi_{1 x x x} \bar{\psi}_{2}-\psi_{1} \bar{\psi}_{2 x x x}-\psi_{1 x x}\right. \\
& \left.\bar{\psi}_{2 x}+\psi_{1 x} \bar{\psi}_{2 x x}\right) \text {, } \\
& \tilde{G}_{13}=i \lambda^{4} \psi_{1}+\lambda^{3} \psi_{1 x}-i \lambda^{2} \psi_{1 x x}+2 i \lambda^{2} \psi_{1} \psi_{2} \bar{\psi}_{2}-3 \lambda\left(\psi_{1 x} \bar{\psi}_{2} \psi_{2}+\psi_{1} \bar{\psi}_{2} \psi_{2 x}\right) \\
& -\lambda \psi_{1 x x x}-4 i\left(\psi_{1 x} \psi_{2}\right)_{x} \bar{\psi}_{2}-2 i\left(\bar{\psi}_{2}\left(\psi_{1} \psi_{2 x}\right)_{x}+\psi_{1}\left(\psi_{2 x} \bar{\psi}_{2}\right)_{x}-\left(\psi_{1} \bar{\psi}_{2 x}\right.\right. \\
& )_{x} \psi_{2}\right)+i \psi_{1 x x x x} \\
& \tilde{G}_{21}=-i \lambda^{3} \psi_{2} \bar{\psi}_{1}-\lambda^{2}\left(\psi_{2 x} \bar{\psi}_{1}-\psi_{2} \bar{\psi}_{1 x}\right)+i \lambda\left(\psi_{2 x x} \bar{\psi}_{1}+\psi_{2} \bar{\psi}_{1 x x}-\psi_{2 x} \bar{\psi}_{1 x}\right) \\
& +2\left(\psi_{2} \bar{\psi}_{1 x} \psi_{1} \bar{\psi}_{1}-\psi_{2} \bar{\psi}_{2} \psi_{2 x} \bar{\psi}_{1}\right)+\left(\psi_{2 x x x} \bar{\psi}_{1}-\psi_{2} \bar{\psi}_{1 x x x}-\psi_{2 x x} \bar{\psi}_{1 x}\right. \\
& \left.+\psi_{2 x} \bar{\psi}_{1 x x}\right) \text {, } \\
& \tilde{G}_{22}=-i \lambda^{5}-i \lambda^{3} \psi_{2} \bar{\psi}_{2}-\lambda^{2}\left(\psi_{2 x} \bar{\psi}_{2}-\psi_{2} \bar{\psi}_{2 x}\right)+i \lambda\left(\psi_{2} \bar{\psi}_{2 x x}+\psi_{2 x x} \bar{\psi}_{2}-\psi_{2 x}\right. \\
& \left.\bar{\psi}_{2 x}\right)+3 i \lambda \psi_{2} \bar{\psi}_{1} \psi_{1} \bar{\psi}_{2}+4\left(\psi_{2} \bar{\psi}_{1} \bar{\psi}_{2 x} \psi_{1}-\psi_{2 x} \psi_{1} \bar{\psi}_{1} \bar{\psi}_{2}\right)-2\left(\bar{\psi}_{1} \psi_{2} \psi_{1 x} \bar{\psi}_{2}\right. \\
& \left.-\psi_{2} \bar{\psi}_{1 x} \bar{\psi}_{2} \psi_{1}\right)+\left(\psi_{2 x x x} \bar{\psi}_{2}-\psi_{2} \bar{\psi}_{2 x x x}-\psi_{2 x x} \bar{\psi}_{2 x}+\psi_{2 x} \bar{\psi}_{2 x x}\right), \\
& \tilde{G}_{23}=i \lambda^{4} \psi_{2}+\lambda^{3} \psi_{2 x}-i \lambda^{2} \psi_{2 x x}-2 i \lambda^{2} \psi_{2} \bar{\psi}_{1} \psi_{1}-3 \lambda\left(\psi_{2} \bar{\psi}_{1} \psi_{1 x}+\psi_{2 x} \bar{\psi}_{1} \psi_{1}\right) \\
& -\lambda \psi_{2 x x x}-4 i\left(\psi_{2 x} \psi_{1}\right)_{x} \bar{\psi}_{1}-2 i\left(\bar{\psi}_{1}\left(\psi_{2} \psi_{1 x}\right)_{x}-\psi_{2}\left(\bar{\psi}_{1} \psi_{1 x}\right)_{x}-\left(\psi_{2} \bar{\psi}_{1 x}\right)_{x}\right. \\
& \left.\psi_{1}\right)+i \psi_{2 x x x x}, \\
& \tilde{G}_{31}=i \lambda^{4} \bar{\psi}_{1}-\lambda^{3} \bar{\psi}_{1 x}-i \lambda^{2} \bar{\psi}_{1 x x}-2 i \lambda^{2} \bar{\psi}_{2} \psi_{2} \bar{\psi}_{1}+3 \lambda\left(\bar{\psi}_{2 x} \psi_{2} \bar{\psi}_{1}+\bar{\psi}_{2} \psi_{2} \bar{\psi}_{1 x}\right) \\
& +\lambda \bar{\psi}_{1 x x x}-4 i\left(\bar{\psi}_{2} \bar{\psi}_{1 x}\right)_{x} \psi_{2}+2 i\left(\bar{\psi}_{1}\left(\bar{\psi}_{2 x} \psi_{2}\right)_{x}+\bar{\psi}_{2}\left(\psi_{2 x} \bar{\psi}_{1}\right)_{x}-\left(\bar{\psi}_{2 x} \bar{\psi}_{1}\right)_{x}\right. \\
& \left.\psi_{2}\right)+i \bar{\psi}_{1 x x x x}, \\
& \tilde{G}_{32}=i \lambda^{4} \bar{\psi}_{2}-\lambda^{3} \bar{\psi}_{2 x}-i \lambda^{2} \bar{\psi}_{2 x x}-2 i \lambda^{2} \bar{\psi}_{1} \psi_{1} \bar{\psi}_{2}+3 \lambda\left(\bar{\psi}_{1 x} \psi_{1} \bar{\psi}_{2}+\bar{\psi}_{1} \psi_{1} \bar{\psi}_{2 x}\right) \\
& +\lambda \bar{\psi}_{2 x x x}-4 i\left(\bar{\psi}_{1} \bar{\psi}_{2 x}\right)_{x} \psi_{1}+2 i\left(\bar{\psi}_{2}\left(\bar{\psi}_{1 x} \psi_{1}\right)_{x}+\bar{\psi}_{1}\left(\psi_{1 x} \bar{\psi}_{2}\right)_{x}-\left(\bar{\psi}_{1 x} \bar{\psi}_{2}\right)_{x}\right. \\
& \left.\psi_{1}\right)+i \bar{\psi}_{2 x x x x}, \\
& \tilde{G}_{33}=-2 i \lambda^{5}+i \lambda^{3}\left(\bar{\psi}_{1} \psi_{1}+\bar{\psi}_{2} \psi_{2}\right)-\lambda^{2}\left(\bar{\psi}_{1 x} \psi_{1}-\bar{\psi}_{1} \psi_{1 x}+\bar{\psi}_{2 x} \psi_{2}-\bar{\psi}_{2} \psi_{2 x}\right) \\
& -i \lambda\left(\bar{\psi}_{1 x x} \psi_{1}+\bar{\psi}_{2 x x} \psi_{2}+\bar{\psi}_{2} \psi_{2 x x}+\bar{\psi}_{1} \psi_{1 x x}-\bar{\psi}_{1 x} \psi_{1 x}-\bar{\psi}_{2 x} \psi_{2 x}\right)-6 \\
& i \lambda \bar{\psi}_{1} \psi_{1} \bar{\psi}_{2} \psi_{2}+6\left(\bar{\psi}_{1} \psi_{1 x} \psi_{2} \bar{\psi}_{2}+\bar{\psi}_{2} \psi_{2 x} \psi_{1} \bar{\psi}_{1}-\bar{\psi}_{2} \bar{\psi}_{1 x} \psi_{2} \psi_{1}-\bar{\psi}_{1} \bar{\psi}_{2 x} \psi_{1}\right. \\
& \left.\psi_{2}\right)+\left(\bar{\psi}_{1 x x x} \psi_{1}-\bar{\psi}_{1} \psi_{1 x x x}-\bar{\psi}_{1 x x} \psi_{1 x}+\bar{\psi}_{1 x} \psi_{1 x x}\right)+\left(\bar{\psi}_{2 x x x} \psi_{2}-\bar{\psi}_{2}\right. \\
& \left.\psi_{2 x x x}-\bar{\psi}_{2 x x} \psi_{2 x}+\bar{\psi}_{2 x} \psi_{2 x x}\right) \text {. }
\end{aligned}
$$

Based on the zero-curvature formulation of $\tilde{F}$ and $\tilde{G}$, we have the fermionic fifth-order NLSE,

$$
\begin{aligned}
& i \psi_{1 t}+4 i\left(\psi_{1 x} \psi_{2}\right)_{x x} \bar{\psi}_{2}+4 i\left(\psi_{1 x} \psi_{2}\right)_{x} \bar{\psi}_{2 x}+2 i\left(\bar{\psi}_{2}\left(\psi_{1} \psi_{2 x}\right)_{x}+\psi_{1}\left(\psi_{2 x} \bar{\psi}_{2}\right)_{x}-\left(\psi_{1} \bar{\psi}_{2 x}\right)_{x}\right. \\
& \left.\psi_{2}\right)_{x}-i \psi_{1 x x x x x}+i \psi_{1}\left(\left(\bar{\psi}_{2 x} \psi_{2}-\bar{\psi}_{2} \psi_{2 x}\right)_{x x}-2\left(\bar{\psi}_{2 x x} \psi_{2 x}-\bar{\psi}_{2 x} \psi_{2 x x}\right)\right)+i\left(\left(\psi_{1 x} \bar{\psi}_{2}-\right.\right. \\
& \left.\left.\psi_{1} \bar{\psi}_{2 x}\right)_{x x}-2\left(\psi_{1 x x} \bar{\psi}_{2 x}-\psi_{1 x} \bar{\psi}_{2 x x}\right)\right) \psi_{2}=0,
\end{aligned}
$$




$$
\begin{aligned}
& i \psi_{2 t}+4 i\left(\psi_{2 x} \psi_{1}\right)_{x x} \bar{\psi}_{1}+4 i\left(\psi_{2 x} \psi_{1}\right)_{x} \bar{\psi}_{1 x}+2 i\left(\bar{\psi}_{1}\left(\psi_{2} \psi_{1 x}\right)_{x}-\psi_{2}\left(\bar{\psi}_{1} \psi_{1 x}\right)_{x}-\left(\psi_{2} \bar{\psi}_{1 x}\right)_{x}\right. \\
& \left.\psi_{1}\right)_{x}-i \psi_{2 x x x x x}+i \psi_{2}\left(\left(\bar{\psi}_{1 x} \psi_{1}-\bar{\psi}_{1} \psi_{1 x}\right)_{x x}-2\left(\bar{\psi}_{1 x x} \psi_{1 x}-\bar{\psi}_{1 x} \psi_{1 x x}\right)\right)-i\left(\left(\psi_{2 x} \bar{\psi}_{1}-\right.\right. \\
& \left.\left.\psi_{2} \bar{\psi}_{1 x}\right)_{x x}-2\left(\psi_{2 x x} \bar{\psi}_{1 x}-\psi_{2 x} \bar{\psi}_{1 x x}\right)\right) \psi_{1}=0 .
\end{aligned}
$$

\section{Summary and Discussion}

In this study, we have discussed the fifth-order HS model with two types of constraints which are $S^{2}=S$ and $S^{2}=3 S-2 I$, respectively. We construct their gauge equivalent equations, which are the super and fermionic fifth-order NLSEs. It is well known there is a close relationship between the HS model and the Hubbard model which attracts lots of interest in physics. This study further demonstrates that the method in this paper can be also applied to establish other higher-order HS models and higher-order super and fermionic NLSE. For the applications of the generalized HS models and higher-order related NLSEs constructed in this paper, we shall try to do it in a near future.

\section{Acknowledgements}

This work is partially supported by National Natural Science Foundation of China (Grant Nos. 11965014, 11605096 and 11601247), We thank Prof. Ke Wu and Prof. Weizhong Zhao (CNU, China) for long-term encouragement and support.

\section{References}

[1] M. Lakshmanan, Continuum spin system as an exactly solvable dynamical system, Phys. Lett. A 61 (1977) 53-54.

[2] V.E. Zakharov and L.A. Takhtadzhyan, Equivalence of the nonlinear Schrödinger equation and the equation of a Heisenberg ferromagnet, Theor. Math. Phys. 38 (1979) 17-23.

[3] G. Ferraz, F.B. Ramos, R. Egger and R.G. Pereira, Spin chain network construction of chiral spin liquids, Phys. Rev. Lett. 123 (2019) 137202.

[4] T. Harmark and M. Orselli, Matching the hagedorn temperature in AdS/CFT correspondence, Phys. Rev. D 74 (2006) 126009.

[5] F.G.S.L. Brandão, E. Crosson, M.B. Sahinoğlu and J. Bowen, Quantum error correcting codes in eigenstates of translation-invariant spin chains, Phys. Rev. Lett. 123 (2019) 110502.

[6] L. Martina, O.K. Pashaev, and G. Soliani, Integrable dissipative structures in the gauge theory of gravity, Class. Quan. Grav. 14 (1997) 3179-3186. 
[7] D.F. Bian, B.L. Guo and L.M. Ling, High-order soliton solution of Landau-Lifshitz equation, Stud. Appl. Math. 134 (2014) 181-214.

[8] L.A. Takhtajan, Integration of the continuous Heisenberg spin chain through the inverse scattering method, Phys. Lett. A 64 (1977) 235-237.

[9] A.B. Yanovski and T.I. Valchev, Pseudo-hermitian reduction of a generalized Heisenberg Ferromagnet equation. i. auxiliary system and fundamental properties, J Nonlin Math Phys 25 (2018) 324-350.

[10] T.I. Valchev and A.B. Yanovski, Pseudo-hermitian reduction of a generalized Heisenberg Ferromagnet equation. ii. special solutions, J Nonlin Math Phys 25 (2018) 442-461.

[11] A. Myrzakul and R. Myrzakulov, Integrable geometric flows of interacting curves/surfaces, multilayer spin systems and the vector nonlinear Schrödinger equation, Int J Geom Methods Mod Phys 14 (2017) 1750136.

[12] R. Myrzakulov, S. Vijayalakshmi, R.N. Syzdykova and M. Lakshmanan, On the simplest $(2+1)$ dimensional integrable spin systems and their equivalent nonlinear Schrödinger equations, J Math Phys 39 (1998) 2122.

[13] R. Myrzakulov, G. Mamyrbekova, G. Nugmanova and M. Lakshmanan, Integrable (2 + 1)-dimensional spin models with self-consistent potentials, Symmetry 7 (2015) 1352-1375.

[14] F. Demontis, S. Lombardo, M. Sommacal, C. van der Mee and F. Vargiu, Effective generation of closed-form soliton solutions of the continuous classical Heisenberg ferromagnet equation, Comm. Nonlin. Sci. Num. Sim. 64 (2018) 35-65.

[15] A. Grekov, A. Zabrodin and A. Zotov, Supersymmetric extension of qKZ-Ruijsenaars correspondence, Nucl. Phys. B 939 (2019) 174-190.

[16] S. Krivonos, O. Lechtenfeld and A. Sutulin, Supersymmetric many-body Euler-CalogeroMoser model, Phys. Lett. B 790 (2019) 191-196.

[17] K. Shimizu, Aspects of massive gauge theories on three sphere in infinite mass limit, J. High Energy Phys. 01 (2019) 090.

[18] A.G. Grau, C. Kristjansen, M. Volk and M. Wilhelm, A quantum check of nonsupersymmetric AdS/dCFT, J. High Energy Phys. 01 (2019) 007.

[19] T. Bargheer, J. Caetano, T. Fleury, S. Komatsu and P. Vieira, Handling handles: nonplanar integrability in $N=4$ supersymmetric Yang-Mills theory, Phys. Rev. Lett. 121 (2018) 231602. 
[20] P. Mathieu, Supersymmetric extension of the Korteweg-de Vries equation, J. Math. Phys. 29 (1988) 2499-2506.

[21] V.G. Makhankov and O.K. Pashaev, Continual classical Heisenberg models defined on graded $s u(2,1)$ and $s u(3)$ algebras, J. Math. Phys. 33 (1992) 2923-2936.

[22] J.F. Guo, S.K. Wang, K. W, Z.W. Yan and W.Z. Zhao, Integrable higher order deformations of Heisenberg supermagnetic model, J. Math. Phys. 50 (2009) 113502.

[23] Z.W. Yan, S.K. Yao, C.H. Zhang and Gegenhasi, The fermionic covariant prolongation structure of the super generalized Hirota equation, Mod. Phys. Lett. B 30 (2016) 1650251.

[24] Z.W. Yan, B. Gao, M.R. Chen and J.F. Cui, On the higher order Heisenberg supermagnet model in (2+1)-dimensions, Chaos Soli. Frac. 118 (2019) 94-105.

[25] Z.W. Yan, M.R. Chen, K. Wu and W.Z Zhao, (2+1)-dimensional integrable Heisenberg supermagnet model, J. Phys. Soc. Jpn. 81 (2012) 094006.

[26] Z.W. Yan, M.N. Zhang, D.Y. Ren, H. Zhang and J.F. Cui, On a super generalized $x$ dependent Hirota equation, Z. Naturforsch. A 72 (2017) 811-815.

[27] Z.W. Yan, M.N. Zhang and J.F. Cui, Higher-order inhomogeneous generalized Heisenberg supermagnetic model, Chin. Phys. Lett. 35 (2018) 050201. 